\section{OPEN ACCESS}

Edited by:

Andrea Martinuzzi,

Eugenio Medea (IRCCS), Italy

Reviewed by:

Yanet Karina Gutierrez-Mercado, Universidad de Guadalajara, Mexico Genaro Pimienta-Rosales, Sanford Burnham Prebys Medical Discovery Institute, United States Hermelinda Salgado-Ceballos,

Mexican Social Security Institute (IMSS), Mexico

*Correspondence: Rachel Sarabia-Estrada Estrada.Rachel@mayo.edu

Specialty section:

This article was submitted to Neurorehabilitation, a section of the journal Frontiers in Neurology

Received: 22 January 2020 Accepted: 16 June 2020 Published: 30 July 2020

Citation: Lara-Velazquez M, Alkharboosh R, Norton ES, Ramirez-Loera $C$ Freeman WD, Guerrero-Cazares $H$,

Forte AJ, Quiñones-Hinojosa A and Sarabia-Estrada R (2020) Chitosan-Based Non-viral Gene and Drug Delivery Systems for Brain Cancer. Front. Neurol. 11:740. doi: 10.3389/fneur.2020.00740

\title{
Chitosan-Based Non-viral Gene and Drug Delivery Systems for Brain Cancer
}

\begin{abstract}
Montserrat Lara-Velazquez ${ }^{1,2}$, Rawan Alkharboosh ${ }^{1,3,4}$, Emily S. Norton ${ }^{1,3,4}$, Cristopher Ramirez-Loera ${ }^{5}$, William D. Freeman ${ }^{1}$, Hugo Guerrero-Cazares ${ }^{1}$, Antonio J. Forte ${ }^{1,6}$, Alfredo Quiñones-Hinojosa ${ }^{1}$ and Rachel Sarabia-Estrada ${ }^{1 *}$
\end{abstract}

${ }^{1}$ Mayo Clinic Florida, Department of Neurosurgery, Jacksonville, FL, United States, ${ }^{2}$ Plan of Combined Studies in Medicine (PECEM), UNAM, Mexico City, Mexico, ${ }^{3}$ Neuroscience Graduate Program, Mayo Clinic Graduate School of Biomedical Sciences, Mayo Clinic, Rochester, MN, United States, ${ }^{4}$ Regenerative Sciences Training Program, Center for Regenerative Medicine, Mayo Clinic, Rochester, MN, United States, ${ }^{5}$ Monterrey Institute of Technology, School of Medicine and Health Sciences, Monterrey, Mexico, ${ }^{6}$ Division of Plastic Surgery and Robert D. and Patricia E. Kern Center for the Science of Health Care Delivery, Mayo Clinic, Jacksonville, FL, United States

Central nervous system (CNS) tumors are a leading source of morbidity and mortality worldwide. Today, different strategies have been developed to allow targeted and controlled drug delivery into the brain. Gene therapy is a system based on the modification of patient's cells through the introduction of genetic material to exert a specific action. Administration of the foreign genetic material can be done through viral-mediated delivery or non-viral delivery via physical or mechanical systems. For brain cancer specifically, gene therapy can overcome the actual challenge of blood brain barrier penetration, the main reason for therapeutic failure. Chitosan (CS), a natural based biodegradable polymer obtained from the exoskeleton of crustaceans such as crab, shrimp, and lobster, has been used as a delivery vehicle in several non-viral modification strategies. This cationic polysaccharide is highly suitable for gene delivery mainly due to its chemical properties, its non-toxic nature, its capacity to protect nucleic acids through the formation of complexes with the genetic material, and its ease of degradation in organic environments. Recent evidence supports the use of CS as an alternative gene delivery system for cancer treatment. This review will describe multiple studies highlighting the advantages and challenges of CS-based delivery structures for the treatment of brain tumors. Furthermore, this review will provide insight on the translational potential of various CS based-strategies in current clinical cancer studies. Specifically, CS-based nanostructures including nanocapsules, nanospheres, solid-gel formulations, and nanoemulsions, also microshperes and micelles will be evaluated.

Keywords: chitosan, brain cancer, brain tumor, nanodelivery, nanoparticles, drug delivery, biodegradable, biomaterials 


\section{INTRODUCTION}

Cancer is the second most frequent cause of mortality following cardiovascular disease, and has surpassed it in high and middleincome countries $(1,2)$. Although primary malignant central nervous system (CNS) tumors account for $2 \%$ of all cancers, they represent a leading cause for morbidity and mortality worldwide. Malignant CNS tumors are the principal cause of death due to solid tumors in children and the third main cause of death in the 15-34 year age-bracket. The most common presentation of a tumor in the brain is due to metastasis, accounting for $40 \%$ of intracranial tumors (3). Overall, CNS cancers represent a therapeutic challenge due to tumor heterogeneity, comprised of multiple distinct sup-populations of cells within the same tumor; each with distinct molecular features and biological responses. Furthermore, genetic and epigenetic alterations affect the progression of the disease as well as response to treatment (4). Treatment of brain tumors includes surgical resection; however, due to the infiltrative nature of some tumors, recurrence at original site and surrounding areas (up to $3 \mathrm{~cm}$ of the margin of the primary lesion) is often seen, and may be the primary cause of poor prognosis (5). Following surgery, a scheme based on chemotherapy and radiation is considered the standard of treatment. However, limited benefits are achieved after this multimodal strategy, mainly driven by poor drug tissue penetration and accumulation in targeted areas (6).

Nano-delivery systems have been shown to be a promising strategy against multiple types of cancer $(7,8)$. The possibility of modulating gene expression or the delivery of specific compounds to regulate different pathways in tumor progression has emerged as a promising alternative for CNS malignancies. Drugs or genes are attached to a variety of compounds, followed by a systemic injection or local administration into the tumor $(9,10)$. Remarkably, the materials show high specificity and tissue penetration in diseased area when injected locally, decreasing systemic toxicity $(11,12)$. Anatomical barriers, such as the blood brain barrier (BBB), are major challenges to drug penetration, often resulting in therapeutic failure (13). Nano-size materials used as vectors may serve to overcome this limitation and effectively deliver therapeutics to site of injury (9).

In CNS cancers, one of the main challenges is the administration of chemotherapeutic agents and the successful action of the drug in the desired area. For brain tumors, selective penetration of the BBB is a limiting factor for successful eradication of cancer cells (14). Chitosan (CS) -nanomaterials are local delivery systems that overcome the limitations imposed by the $\mathrm{BBB}$, and allow sustained, controlled and prolonged drug release in specific areas, decreasing the risk of systemic toxicity (15). These systems can also be used for real time tracking of cancer cells when acting as imaging probes for various imaging techniques (fluorescent image guiding, magnetic resonance imaging (MRI), computed tomography (CT), positron-emission tomography (PET) and optical imaging) (16). For diagnostic purposes, when compared with free drugs, CS-nanomaterials increase the stability of contrast enhancing agents and drugs with specific accumulation in target areas. Due to their compact size and protein surface interactions, CS-nanostructure components are able to travel in small blood vessels throughout the body. Upon arrival to the tumor area, CS-nanostructures leave the systemic blood flow through disrupted tumor vasculature, and are concentrated and retained in the tumor area (tumor -homing effect) (17).

In this review, we will highlight the recent advances in CSbased gene and drug delivery systems using nanotechnology for the treatment of brain cancer.

\section{BRAIN TUMORS}

Brain tumors are one of the most devastating types of cancer, with the most malignant form having a median survival of $\sim 15$ months. Brain tumors can be primary, meaning they arise from the native cells of the brain, or they can be metastatic, arising from tumors that have spread from other organs. Brain tumors have an annual incidence of about 22 people per 100,000 in the United States, with incidence increasing with age (18). Interestingly, brain tumors as a whole occur more frequently in women, while malignant brain tumors are more common in men, indicating a sex difference in brain tumor biology (18). Out of adult primary brain tumors, approximately one-third are malignant (19). Tumors are typically diagnosed through combined neurological exams, MRI of the brain, CT and PET scans to determine whether the tumor is a metastasis arising from another site in the body, and through tumor biopsy (20).

Gliomas, or tumors arising from glial cells, account for over $75 \%$ of malignant adult brain tumors. These tumors are classified by the World Health Organization (WHO) by histopathological features and molecular findings. Diffuse gliomas can be stratified by their cell origin through histological characterization (21). The cell of origin is controversial, with various research studies citing neural stem cells as the source of origin, while others cite glial progenitors; classifications are based on features of glial cells. Astrocytomas present features of astrocytes, the star-shaped glial cells important for brain homeostasis, while oligodendrogliomas express features of oligodendrocytes, the cells that produce myelin. Anaplastic astrocytomas and glioblastomas represent $38 \%$ of primary brain tumors $(3,22)$. Diffuse gliomas are classified by the WHO as oligodendrogliomas (grade II), anaplastic oligodendroglioma (grade III), diffuse astrocytoma (grade II), anaplastic astrocytoma (grade III), and the most common glioblastoma (GBM) (grade IV) (21). Gliomas are further defined by their isocitrate dehydrogenase (IDH) $1 / 2$ mutation status. Mutations in IDH 1 and 2 are extremely common in low grade gliomas and secondary high grade gliomas, or high grade tumors that progress from lower grade tumors (23). However, this mutation is relatively rare in primary GBM (23). Additionally, 1p/19q co-deletion, ATRX loss, and TP53 mutation is profiled in order to fully define diffuse gliomas (21). GBM can be further characterized into four molecular subtypesproneural, neural, classical, and mesenchymal-based on distinct transcriptional signatures (24).

The current standard of care for GBM involves a combinatorial strategy of surgical resection, chemotherapy, and radiation treatment (25). The addition of the chemotherapeutic 
drug, temozolomide (TMZ), to the treatment strategy in 2005 increased median survival of patients from 12.1 to 14.6, signifying the last major change to GBM treatment (25). Recent medical advances including the development of tumor treating fields via the Optune ${ }^{\circledR}$ system have also shown a significant survival benefit, although these treatments do not provide a cure for GBM (26). The current status of brain tumor management results in a significant need for the development of better therapeutic options to improve patient care (6).

\section{NON-VIRAL MEDIATED DELIVERY SYSTEMS}

In contrast to viral vectors, non-viral delivery systems are better tolerated, can carry large amounts of nucleic acid and have a higher safety index due to their transient expression compared to stable modifications (27). CS is an organic molecule that is less toxic than other cationic polymers such as polyethyleneimine, polylysine, or polyarginine (27), and is therefore a promising excipient for non-viral gene and drug delivery systems. Non-viral delivery can be divided into physical or chemical methods (27-30).

\section{Physical Delivery Systems}

Electroporation: An electrical pulse is applied to the cells to increase the permeability of the cell membrane facilitating uptake of DNA strands (31).

Direct injection of nucleic acids: This method has shown a relative degree of success in some tissues, however, without protection following systemic injection, the plasmid DNA (pDNA) is rapidly broken down by nucleases (31).

\section{Chemical Delivery Systems}

Cationic lipids: Lipid-based systems such as FuGene, GenePORTER, Transfast, DOTAP, and Lipofectamine 2000TM are commercially available lipid-based vectors. They are positively charged and encapsulate the anionic nucleic acid to enable cell entry via endocytosis. Lipofectamine 2000TM is the most commonly used reagent and often acts as a positive control in many studies (32).

Cationic polymers: These polymers are positively charged materials that bind electrostatically to negatively charged nucleic acid to form delivery vectors (33). Polymers such as Poly (Llysine) (PLL), polyethyleneimine (PEI), and Polyamidoamine (PAMAM) dendrimers, have shown promising results preclinically, however, toxicity and side effects are often displayed in vivo and in vitro experiments, ultimately limiting their translational potential (34-36).

Complexation with nucleic acid can reduce the charge of synthetic polymers, for that reason, there is growing concern regarding the degradation and ultimate fate of the construct of non-viral vectors. There is a growing interest in the use of natural biocompatible and biodegradable polymers such as CS (37) which has been used extensively in nucleic acid delivery. CS meets the criteria for a successful non-viral nucleic acid delivery carrier: efficiency in cell uptake, protection of nucleic acids from degradation, efficient unpacking of the genetic cargo, escape from endosomal pathways, and nuclear import (38).

\section{BIODEGRADABLE POLYMER: CHITOSAN}

Chitosan is the main derivative of chitin (poly-N-acetyl glucosamine), a linear polysaccharide highly biodegradable and one of the most abundant polymers in nature (second only to cellulose) (11). Partial deacetylation in alkaline conditions of chitin results in the production of CS, a positively charged polysaccharide highly soluble in low $\mathrm{pH}$ solutions and poorly soluble in physiological aqueous solutions. CS is present in the exoskeletons of crustaceans (like crabs, lobsters and shellfish), insects and the cellular walls of mycelial fungi with a molecular weight ranging from low $(<100 \mathrm{KDa})$ up to high (>300 KDa) (39). This biomaterial is non-toxic, biocompatible and biodegradable with low allergenicity. It also functions as an antioxidant, hemostatic agent (40, 41); and chelator of elements such as iron, copper and magnesium. CS is cleared by enzymatic hydrolysis mediated by intestinal microorganisms and lysozymes. The main derivatives with medical applicability are N,N,N-trimethyl-CS, N,Ocarboxymethyl-CS and O-carboxymethyl-N,N,N-trimethyl-CS $(39,42)$.

Due to its ability to modulate the inflammatory response, CS has been used for the repair of damaged tissue (woundhealing) by promoting formation of granular tissue after injury (40). Additionally, CS increases the action of neutrophils, macrophages and fibroblasts, ultimately speeding the process of tissue repair. The tissue repair effects of CS are dependent on molecular weight, degree of chemical modification (deacetylation), and CS presentation. Therefore, CS has unique properties that could enhance neuroregeneration by mitigating secondary neuroinflammatory tissue injury. Another strategy for wound healing treatment is through CS-mediated vehicles to deliver growth factors (i.e., FGF, EGF), this option allows for an extended action of the growth factor in the desired location (42).

Antimicrobial action of CS is mediated by its cationic charge that destabilizes the negative bacteria cell membrane, leading to a leakage of inner cellular components (proteins, nucleic acids) and increased permeability in the bacteria cellular membrane impairing nutrient uptake (43). Interestingly, lower concentrations of CS $(<0.2 \mathrm{mg} / \mathrm{ml})$ cause bacterial agglutination, while higher concentrations keep them in suspension (40). This biomaterial has broad potency against gram-positive and negative bacteria such as $S$. aureus, $P$. aeruginosa, $P$. mirabilis, and E. Coli (43); causing osteomyelitis, cystitis, periodontitis, mucositis, burn, and skin infections among others. The potency of CS biomaterial is dependent on the dose, $\mathrm{pH}$ and temperature and on the composition of the polymer (hydrogels, coatings, powder, solution, films, pure, or loaded with different materials) (44). 


\section{CHITOSAN-BASED NANOSTRUCTURES FOR BRAIN CANCER TREATMENT}

CS multifunctionality and high cargo entrapment efficiency make CS derivatives versatile nanodelivery vehicles. Chitin monomers are linearized under alkaline conditions by deacetylation in the solid state or by the enzymatic hydrolysis of chitin deacetylases (45). The bipolyaminosaccharide structure is composed of a carbohydrate backbone and abundant $-\mathrm{OH}$ and $-\mathrm{NH}_{2}$ functional groups that act as readily accessible moieties for functional modifications. This enables tuning for efficient crosslinking, controlled drug release profile, enhanced electrostatic interaction, and increased solubility. The degree of deacetylation and molecular weight ratio of CS-nitrogen to phosphatecargo make CS a suitable biomaterial that could be utilized for nanoparticle synthesis and nanomaterial fabrication for the delivery of therapeutic agents (46). Amongst the most common nanodelivery systems explored, CS nanoparticles (NPs) have provided a great degree of safety and durability across various pharmaceutical and pre-clinical applications. The inherent cationic nature of CS allows efficient binding to microtubules or motor proteins for cytoplasmic trafficking, increased plasmid or cargo release efficiency mediated by the osmotic pressure in the endosome (caused by influx of hydrogen protons), and finally, low toxicity index due to its biocompatibility and biodegradability across various biological applications (46). Cargo is either complexed or confined inside the CS particle, or dispersed in a CS matrix. These particulate systems can be prepared by cross-linking, cationic salts solvation, emulsification, ionic complexation, or gelation methods by reacting with different functional groups on proteins, antibodies, drugs, DNA/RNA or other $\mathrm{pH}$ sensitive moieties (46).

Particles are characterized by their spherical diameter and spatial composition, with microparticles/microspheres ranging between 1 and $1,000 \mu \mathrm{m}$ and NPs/nanospheres measuring between $1 \mathrm{~nm}$ and $>1 \mu \mathrm{m}$ (47). NPs (and microparticles) are characterized by their constituent components and can be referred to as "nanocapsules," a vesicular particulate system with a hollow sphere consisting of an oil or water core (that may include active cargo), and a polymeric shell (48). This structure mediates cargo entrapment in the core or adsorption on the particle surface. Conversely, matricial structures are referred to as "nanospheres" and denote particulate systems where the active molecule is incorporated into the polymer network (48). Moreover, "solid-lipid" NPs refer to systems that utilize lipids in the solid phase and subsequent emulsification with a surfactant for structure stability (49). This structure is advantageous when delivering cargo that is poorly water soluble. In line with lipid structures are "nanoemulsions" created by the mixture of two immiscible liquids stabilized by a surfactant. Single lipid layer derived particles are referred to as "micelles" and do not contain an aqueous core (50). Table 1 summarizes the advantages and disadvantages of the described nanostructures.

CS nanosystems are selected based on multiple factors including cargo polarity, solubility, weight, and rout for optimal administration. CS-coated or CS-formulated particulate systems have proven to be efficient nanocarriers to the CNS. Due to their enhanced membrane adhesive nature, particles carrying genes of interest enable enhanced transfection efficiency to recipient cells. Size and composition of CS nanoparticles are fundamental factors that determine targeting and biodistribution to tumors of various origins (55). Nanosized carriers are suitable for disease models that are hypervascularized, such as brain tumors, and would benefit from the enhanced permeability and retention effect permitting passive diffusion in the intratumoral space; this effect limits off-target toxicity (56).

While the development of CS-based nanocarrier technology for brain tumors have primarily focused on the encapsulation and delivery of chemotherapeutics, we will attempt to highlight current advances in non-viral gene delivery strategies using CS nanoparticles along with some promising strategies of drug and chemotherapeutic based encapsulation approaches used for brain cancer treatment.

TABLE 1 | Advantages and disadvantages of distinct nanostructures.

\begin{tabular}{|c|c|c|c|}
\hline Morphology & Advantages & Disadvantages & References \\
\hline Nanocapsules & $\begin{array}{l}\text { Rapid absorption of cargo and longer retention time at target site. Low } \\
\text { polymer content required for comparable drug loading. Shell prevents } \\
\text { direct contact of cargo with environment offering enhanced protection } \\
\text { of load from degradation }\end{array}$ & $\begin{array}{l}\text { Aggregation of particles and leakage of } \\
\text { cargo }\end{array}$ & (11) \\
\hline Nanospheres & $\begin{array}{l}\text { Slow and sustained release of encapsulated cargo. Higher efficiency } \\
\text { and low toxicity. More readily protects cargo against reticuloendothelial } \\
\text { system }\end{array}$ & $\begin{array}{l}\text { Storage by freezing leads to microfibers. } \\
\text { Harsh processing conditions required for } \\
\text { scaled-up manufacturing }\end{array}$ & (51) \\
\hline Nanoemulsions & $\begin{array}{l}\text { Oil droplet protect cargo from oxidation and hydrolysis in circulation. } \\
\text { Efficient self-assembly and solubilization of lipophilic drugs }\end{array}$ & $\begin{array}{l}\text { Rapid release, low stability and lower } \\
\text { encapsulation of hydrophilic molecules }\end{array}$ & (52) \\
\hline Micelles & $\begin{array}{l}\text { Brain cancer targeting moieties to the vasculature widely studied } \\
\text { (transferrin receptor integrins) (53) } \\
\text { RGD peptides, LRP1 (LDL Receptor Related Protein 1) }\end{array}$ & $\begin{array}{l}\text { Non-modified micelles display impaired } \\
\text { penetration through the BBB } \\
\text { (sub-therapeutic delivery of treatment load) }\end{array}$ & (54) \\
\hline
\end{tabular}


TABLE 2 | Multiple chitosan applications in cancer drug delivery.

\begin{tabular}{|c|c|c|c|c|}
\hline Disease model & Morphology & Composition & Preparation & References \\
\hline C6 glioma cells & Nanoemulsions & Polyethylene glycol & $\begin{array}{l}\text { Docetaxel loaded D- } \alpha \text {-tocopherol polyethylene } \\
\text { glycol succinate 1,000 conjugated CS }\end{array}$ & (79) \\
\hline RPMI 2,650 human nasal cell line & Nanocapsules & Lipid-core nanocapsules coated with CS & $\begin{array}{l}\text { Simvastatin- loaded poly- } \varepsilon \text {-caprolactone } \\
\text { nanocapsules coated with CS }\end{array}$ & $(80)$ \\
\hline C6 glioma cells & Nanoemulsions & $\begin{array}{l}\text { Oil kaempferol (KPF) }(0.1 \% \mathrm{w} / \mathrm{w}) \text { in } 16 \% \\
(\mathrm{w} / \mathrm{w}) \text { medium-chain triglyceride }(\mathrm{MCT}) \text { and } \\
5.0 \%(\mathrm{w} / \mathrm{w}) \text { egg-lecithin }\end{array}$ & $\begin{array}{l}\text { KPF-loaded nanoemulsion and KPF-loaded } \\
\text { mucoadhesive nanoemulsion }\end{array}$ & $(74)$ \\
\hline GBM & $\begin{array}{l}\text { Scaffolds- } \\
\text { polyelectrolyte } \\
\text { complexes }\end{array}$ & CS -polyelectrolyte complex scaffolds & Porous CS- scaffolds & $(81)$ \\
\hline Human brain cancer stem cells & NPs & CS-PLGA NPs modified with sialic acid (SA) & Curcumin -loaded CS- PLGA NPs modified with SA & $(82)$ \\
\hline $\begin{array}{l}\text { T98G human GBM cell line and } \\
\text { human umbilical vein endothelial } \\
\text { cells }\end{array}$ & Nanoemulsion & PLGA NPs (50:50) & $\begin{array}{l}\text { 5-FU PLGA (50:50) NPs, bevacizumab, were loaded } \\
\text { into the scaffold }\end{array}$ & (83) \\
\hline GBM & Polymeric NPS & Glycol CS and dextran sulfate NPs & $\begin{array}{l}\text { Methotrexate-loaded polymeric NPs based on } \\
\text { Glycol CS and dextran sulfate }\end{array}$ & (84) \\
\hline $\begin{array}{l}\text { Human brain cancer cell line } \\
\text { (Hs683) }\end{array}$ & $\begin{array}{l}\text { Piperine } \\
\text { micellization }\end{array}$ & Nanomicelles forming core-shell NPs & Optimum piperine-loaded core-shell NPs & (85) \\
\hline C6 glioma cells & NPs & Glycol CS NPs & MTX-loaded CS NPs & (86) \\
\hline Mouse fibroblast cell lines L929 & NPS & Core-shell polymeric NPs & Docetaxel-loaded NPs & $(87)$ \\
\hline
\end{tabular}

\section{CHITOSAN-BASED DELIVERY-SYSTEMS TO BRAIN CANCER}

Treatment-resistant brain tumors, such as grade IV gliomas, overexpress epidermal growth factor receptor (EGFR) and galectin-1, leading to chemotherapy resistance. Amplification of EGFR is found in $>50 \%$ of GBM cases and presents a logical molecular target for GBM therapy (57). Given the importance of EGFR and its isoforms in brain tumors, several agents have undergone clinical trials in an attempt to target EGFR (i.e., lapatinib, gefitinib) but outcomes have been largely disappointing (58). This is partly due to poor BBB penetration, and the discovery that EGFR receptor blockade is not enough to inhibit downstream signaling, suggesting that EGFR receptor blockade may be activating other pathways that confer cell survival (59). Such a phenomenon would benefit from directed gene silencing. In an effort to examine the silencing efficiency of EGFR and Galectin-1 in U87 human GBM line, CS lipid nanocapsules were complexed with anti-EGFR and anti-galactin-1 small interfering RNA (siRNA) and administered via convection enhanced delivery (CED) (a minimally invasive surgery that placed catheters directly into the tumor bed to deliver pharmaceutical agents) in athymic nude mice (60). Treated groups received concomitant TMZ administration to examine chemotherapy resistance or response after gene silencing. CS nanocapsules carrying EGFR and galectin-1 siRNA significantly increased survival in tumor-bearing mice and decreased gene expression in tumor tissue. While CED administration proved effective in the delivery of CS nanocapsules, another advantage of CS is its mucosal adhesion offering a different administration route. Intranasal delivery has gained momentum in human clinical trials for therapeutic delivery, partly due to its reduced invasiveness and toxicity. CS nanocapsules were delivered intranasally for RNA interference (RNAi) mediated knockdown of galectin-1 in GL261 mouse glioma line, and demonstrated successful nose-to-brain transport of siRNA along with survival benefits when delivered with programmed cell death-1 (PD-1) immunotherapy in vivo (61). Data suggests that CS based nanocapsules could effectively translocate across the BBB and deliver nucleic acids to brain cancer in vitro and in vivo $(62,63)$.

To circumvent drug delivery limitations to the CNS, poly(lactic-co-glycolic acid) (PLGA) modified CS nanoparticles (CSNPs) were conjugated with Arg-Gly-Asp RGD-linked peptide and loaded with clinically approved paclitaxel (PTX) chemotherapeutic drug for GBM therapy. The PTX-PLGACSNP-RGD particle, prepared by emulsion-solvent evaporation, displayed optimal tumor targeting and uptake via integrin receptor mediated endocytosis, induced cell-cycle arrest at G2/M, and increased lung tumor cell death (64). While authors did not address brain tumors in the study, the incorporated RGD linked ligand targets $\alpha_{\mathrm{v}} \beta_{3}$ integrins on endothelia and is highly expressed in many tumor vasculature beds but largely absent in normal tissue. Hyper-vascularized tumors, such as brain cancers, would benefit from nano-platforms that incorporate integrin targeting strategies for gene or drug delivery. To bypass limitations of chemotherapeutic delivery to the $\mathrm{BBB}$, dual functionalized liposomes were developed to mitigate transportation of doxorubicin and erlotinib to tumor cells. Liposomes were surface modified with transferrin enabling their translocation across endothelial cells lining the blood vessels, and whose surface exhibits high transferrin receptor expression. Additionally, a cell penetrating PFVYLI peptide was coated on the surface to enhance liposomal uptake by U87 human GBM commercial cell line. GBM cells were seeded in PLGA-chitosan 


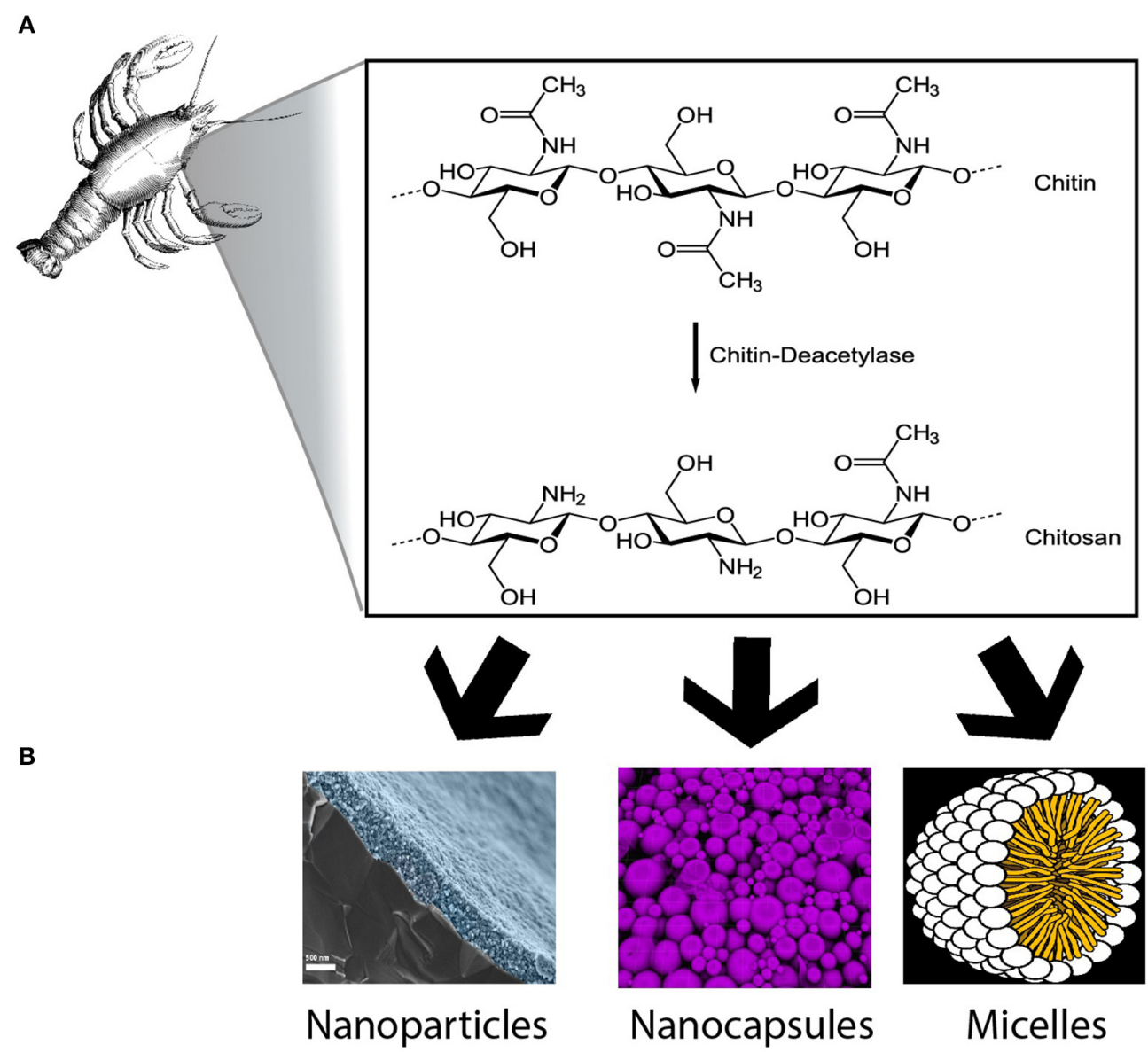

FIGURE 1 | Chitosan based formulations for brain cancer. (A) Representation of the chemical conversion from chitin to chitosan. (B) Nanoparticles, nanocapsules, and micelles are some examples of chitosan formulations described in this review (Images from A,B were obtained from wikimedia commons without any modifications. Chitosan synthesis image by neurotiker, nanoparticles image design by HiguchiJu, nanocapsules image by Stephan Weiss and micelles image by Mariana Ruiz Villareal).

scaffold serving as an in vitro porous scaffold $3 \mathrm{D}$ platform to study the functional translocation and cellular uptake of coated liposomes. Tumor cells seeded in the PLGA-chitosan scaffold resulted in $52 \%$ cell death. This study offers a 3D based platform that acts as a sufficient surrogate to study nanoparticle uptake and translocation in 3D models of brain tumors (65).

Brain targeted chitosan-coated nanoparticles is further shown to enhance particle uptake by human blood-brain barrier cerebral microvessel endothelial cells (hCMECs) via receptor mediated endocytosis. Further evaluation into the mechanisms enabling this translocation revealed a preferential cellular uptake pathway implicating the transferrin receptor with subsequent nanoparticle internalization via receptor-mediated endocytosis (66).

Improved in vivo brain pharmacokinetics of conventional GBM chemotherapy, such as TMZ, was shown to be significantly enhanced when polyamidoamine (PAMAM) dendrimer is coated with chitosan and conjugated to TMZ. Chitosan-coated PAMAM conjugated to TMZ improved GBM tumor targeting in U-251 and T-98G cell lines at lower TMZ concentrations. In vivo pharmacokinetics exhibited sustained release with a half-life of
$22.74 \mathrm{~h}$ in chitosan-coated dendrimer compared to free drug (TMZ alone) at $15.35 \mathrm{~h}$. Reported work revealed that chitosananchored nanoparticles are sufficient at delivering chemotherapy across the BBB and enhanced tumor cell cytotoxicity ex vivo (67).

Similarly, CS nanospheres were constructed by complexing pDNA with CS tripolyphosphate (TPP) and hyaluronic acid (HA) via ionotropic gelation. Ionic gelation permits the formation of sol-gel transition, and TPP stabilizes the complex in biological fluids and decreases particle size (68). Resultant nanosphere (CS-TPP/HA) was evaluated in vitro for intracellular delivery of Pseudovirus (pSV)-luciferase (surrogate gene) to neural stem cells and spinal cord slices along with direct injection into the spinal cord in vivo. HA signals through CD44 and the receptor for hyaluronan mediated motility (RHAMM) on neural stem cells, regulating proliferation and angiogenesis and mediating the radial migration of spinal cord neurons. CS-TPP/HA resulted in higher gene transfection efficiency, less toxicity, and more retention time of CS nanosphere in vitro and in vivo compared to PEI or naked-DNA alone, suggesting a viable carrier for gene delivery to neural stem cells using CS nanospheres. 
Retinoic acid (RA), a derivative of vitamin A, activates Notch signaling response pathways in glioma initiating stem cells, prompting lineage specific differentiation and arrest at the G0/S phase (69). Strategies to induce cancer stem cell differentiation have been widely used across various malignancies, such as the delivery of bone morphogenetic protein 4 (BMP4) to brain tumor initiating cells for astrocytic induction, rendering cancer stem cells more susceptible to chemotherapy (70). Trimethylated solid-lipid CS formulation was constructed for RA encapsulation to evaluate affinity and delivery of RA to U87 human GBM line (70). N-N-N-trimethyl CS-functionalized (TMC) particles offer increased solubility above native CS solubility threshold $(\mathrm{pH}<5.6)(71)$. The polyelectrolytic cationic nature of TMC improves aqueous solubility across a range of $\mathrm{pHs}$ while maintaining efficient cell targeting. TMC solid-lipid particles exhibited significant anticancer effects by inducing apoptosis mediated by the delivery of RA, compared to free RA alone. Trimethylated solid-lipid particles offered enhanced protection from the "burst-effect" and prolonged circulation. Modified solid-lipid CS particles hold great promise for cancer therapy as they can deliver sufficient therapeutic pay-loads, entrap hydrophobic drugs at larger concentrations, and improve drugrelease profile.

Nanoemulsions are the product of the mixing of two immiscible liquids into a single phase through the use of a surfactant (72). The resulting size of emulsified spheres lies between 10 and $1,000 \mathrm{~nm}$ (72). CS has emerged as an attractive coating in nanoemulsions as a way to treat cancer. In particular, CS nanoemulsions have been used to deliver chemotherapeutics in order to increase drug stability, bioavailability of hydrophobic molecules, or drug uptake using positively charged CS to pass the negatively charged biological membranes. In experiments designed to treat brain cancer, the chemotherapeutic 5fluorouracil (5-FU) has been entrapped into a CS nanoemulsion in order to increase uptake (73). The created nanoemulsion retained more $5-\mathrm{FU}$ within the core matrix of resulting particles, resulting in a slow-release profile from nanoemulsion over a period of 30 days (73). Despite encouraging results on drug release, this study did not perform any results on glioma cell viability. Other studies have found incorporating CS into a 5-FU nanoemulsion increases mucoadhesive properties, contributing to the feasibility of intranasal application (74). Adding CS to the nanoemulsion in this case increased mucoadhesion and resulted in increased drug release in vivo in rats (74). Additionally, the 5-FU-loaded CS nanoemulsion resulted in decreased viability and increased apoptosis in C6 rat glioma cells (74), suggesting this may be a practical alternative way to treat glioma. Based on the results found with increased uptake and slow drug release in chemotherapy-loaded CS nanoemulsions, there is high potential for using these methods in DNAbased therapeutics.

Micelles are particles $10-100 \mathrm{~nm}$ in diameter formed by amphiphilic molecules self-assembling by turning hydrophobic compartments inward and hydrophilic compartments outward in solution (75). Similar to nanoemulsions, the addition of CS to micelles is appealing as it allows for increased uptake and bioavailability of hydrophobic compounds. Additionally, the loading of therapeutics into micelles with CS may allow for increased transport across the BBB due to the small, amphiphilic nature of the particles. The use of CS micelles in targeting brain cancer has been limited to the use of chemotherapeutics, but has shown to be relatively effective in drug delivery. When CS-containing micelles are loaded with all-trans RA, there is slow drug release and a significant decrease in the migration of U87 GBM cells compared to the application of free drug (76). Similarly, when loaded with a water-insoluble chemotherapeutic, myricetin, there is increased drug uptake, decreased cell viability and increases apoptosis in vitro (77). The use of CS micelles with myricetin also decreased tumor growth in vivo compared to free drug and controls (77). Adding conjugated CS to micelles can also target glioma cells based on overexpressed receptors on the cell surface. Conjugating CS to d- $\alpha$-tocopheryl glycol succinate 1000 (TPGS) and incorporating it into docetaxelloaded micelles allows for targeting to the transferrin receptors on glioma cells (78). This method of targeting glioma cells for chemotherapy is over 200-fold more effective on C6 rat glioma cell viability than free Docel and also exhibits increased cell uptake and stability in vivo over time (78). Based on the findings with CS-containing micelles in chemotherapy delivery, this could be a potential future avenue for DNA technology in CNS cancer.

Table 2 summarizes some examples of CS-based formulations used as a cargo for chemotherapeutic delivery against cancer.

In conclusion, CS has been widely used in several health care materials and extensively studied; chitosan-coated material may offer novel and improved approaches toward the delivery of cancer therapeutics (88). Its biocompatibility and intrinsic characteristics makes it a suitable option to be used as a carrier for brain cancer therapy. Additionally, it is highly available in nature and represents a cost-effective biomaterial for chemotherapeutic delivery to the brain. Based on many pre-clinical studies detailed above, we anticipate that CS will become widely used in upcoming clinical trials and therapeutic development, particularly as a vehicle for previously approved medications and novel DNA gene therapy targeting brain cancer (Figure 1).

\section{AUTHOR CONTRIBUTIONS}

ML-V, RA, EN, CR-L, and RS-E contributed conception and design of the study. ML-V, RA, EN, and CR-L wrote the first draft of the manuscript and sections of the manuscript. HG-C, AQ-H, WF, and AF contributed reviewing the manuscript. All authors contributed to manuscript revision, read, and approved the submitted version.

\section{FUNDING}

This work was supported by Mayo Clinic Jacksonville, FL. Intramural Funding from the Neurosurgery Department (Convergence).

\section{ACKNOWLEDGMENTS}

ML-V thanks PECEM, UNAM, and CONACYT for the support provided. 


\section{REFERENCES}

1. Kochanek KD, Murphy SL, Xu J, Arias E. Deaths: Final Data for 2016. National Vital Statistics Reports. National Center for Health Statistics (2018).

2. Dagenais GR, Leong DP, Rangarajan S, Lanas F, Lopez-Jaramillo P, Gupta R, et al. Variations in common diseases, hospital admissions, and deaths in middleaged adults in 21 countries from five continents (PURE): a prospective cohort study. Lancet. (2019) 395:785-94. doi: 10.1016/S0140-6736(19)32007-0

3. Buckner JC, Brown PD, O'Neill BP, Meyer FB, Wetmore CJ, Uhm JH. Central nervous system tumors. Mayo Clin Proc. (2007) 82:1271-86. doi: $10.4065 / 82.10 .1271$

4. Fuller CE, Jones DTW, Kieran MW. New classification for central nervous system tumors: implications for diagnosis and therapy. Am Soc Clin Oncol Educ Book. (2017) 37:753-63. doi: 10.14694/EDBK_175088

5. Kirkpatrick JP, Sampson JH. Recurrent malignant gliomas. Semin Radiat Oncol. (2014) 24:289-98. doi: 10.1016/j.semradonc.2014.06.006

6. Lara-Velazquez M, Al-Kharboosh R, Jeanneret S, Vazquez-Ramos C, Mahato $\mathrm{D}$, Tavanaiepour $\mathrm{D}$, et al. Advances in brain tumor surgery for glioblastoma in adults. Brain Sci. (2017) 7:166. doi: 10.3390/brainsci7120166

7. Birk HS, Han SJ, Butowski NA. Treatment options for recurrent high-grade gliomas. CNS Oncol. (2017) 6:61-70. doi: 10.2217/cns-2016-0013

8. Bottai G, Truffi M, Corsi F, Santarpia L. Progress in nonviral gene therapy for breast cancer and what comes next? Expert Opin Biol Ther. (2017) 17:595-611. doi: 10.1080/14712598.2017.1305351

9. Kim JW, Chang AL, Kane JR, Young JS, Qiao J, Lesniak MS. Gene therapy and virotherapy of gliomas. Prog Neurol Surg. (2018) 32:112-23. doi: $10.1159 / 000469685$

10. Choudhury SR, Hudry E, Maguire CA, Sena-Esteves M, Breakefield XO, Grandi P. Viral vectors for therapy of neurologic diseases. Neuropharmacology. (2017) 120:63-80. doi: 10.1016/j.neuropharm. 2016.02.013

11. Bernkop-Schnurch A, Dunnhaupt S. Chitosan-based drug delivery systems. Eur J Pharm Biopharm. (2012) 81:463-9. doi: 10.1016/j.ejpb.2012.04.007

12. Bonadio J, Smiley E, Patil P, Goldstein S. Localized, direct plasmid gene delivery in vivo: prolonged therapy results in reproducible tissue regeneration. Nat Med. (1999) 5:753-9. doi: 10.1038/10473

13. Papademetriou IT, Porter T. Promising approaches to circumvent the bloodbrain barrier: progress, pitfalls and clinical prospects in brain cancer. Ther Deliv. (2015) 6:989-1016. doi: 10.4155/tde.15.48

14. Rossignol J, Srinageshwar B, Dunbar GL. Current therapeutic strategies for glioblastoma. Brain Sci. (2019) 10:15. doi: 10.3390/brainsci100 10015

15. Patel TR. Nanocarrier-based therapies for CNS tumors. CNS Oncol. (2014) 3:115-22. doi: $10.2217 / \mathrm{cns} .14 .2$

16. Key J, Leary JF. Nanoparticles for multimodal in vivo imaging in nanomedicine. Int J Nanomed. (2014) 9:711-26. doi: 10.2147/IJN.S53717

17. Lee JH, Jang JT, Choi JS, Moon SH, Noh SH, Kim JW, et al. Exchange-coupled magnetic nanoparticles for efficient heat induction. Nat Nanotechnol. (2011) 6:418-22. doi: 10.1038/nnano.2011.95

18. Ostrom QT, Cioffi G, Gittleman H, Patil N, Waite K, Kruchko C, et al. CBTRUS statistical report: primary brain and other central nervous system tumors diagnosed in the United States in 2012-2016. Neuro Oncol. (2019) 21:v1-100. doi: 10.1093/neuonc/noz150

19. Lapointe S, Perry A, Butowski NA. Primary brain tumours in adults. Lancet. (2018) 392:432-46. doi: 10.1016/S0140-6736(18)30990-5

20. Abd-Ellah MK, Awad AI, Khalaf AAM, Hamed HFA. A review on brain tumor diagnosis from MRI images: practical implications, key achievements, lessons learned. Magn Reson Imaging. (2019) 61:300-18. doi: 10.1016/j.mri.2019.05.028

21. Louis DN, Perry A, Reifenberger G, von Deimling A, Figarella-Branger D, Cavenee WK, et al. The 2016 World Health Organization classification of tumors of the central nervous system: a summary. Acta Neuropathol. (2016) 131:803-20. doi: 10.1007/s00401-016-1545-1

22. American Cancer Society. Cancer Facts \& Figures 2020. Atlanta, GA: American Cancer Society (2020).

23. Yan H, Parsons DW, Jin G, McLendon R, Rasheed BA, Yuan W, et al. IDH1 and IDH2 mutations in gliomas. N Engl J Med. (2009) 360:765-73. doi: 10.1056/NEJMoa0808710
24. Verhaak RG, Hoadley KA, Purdom E, Wang V, Qi Y, Wilkerson MD, et al. Integrated genomic analysis identifies clinically relevant subtypes of glioblastoma characterized by abnormalities in PDGFRA, IDH1, EGFR, and NF1. Cancer Cell. (2010) 17:98-110. doi: 10.1016/j.ccr.2009.12.020

25. Stupp R, Mason WP, van den Bent MJ, Weller M, Fisher B, Taphoorn MJ, et al. Radiotherapy plus concomitant and adjuvant temozolomide for glioblastoma. N Engl J Med. (2005) 352:987-96. doi: 10.1056/NEJMoa043330

26. Kinzel A, Ambrogi M, Varshaver M, Kirson ED. Tumor treating fields for glioblastoma treatment: patient satisfaction and compliance with the second-generation optune((R)) system. Clin Med Insights Oncol. (2019) 13:1179554918825449. doi: 10.1177/1179554918825449

27. Yu H, Chen X, Lu T, Sun J, Tian H, Hu J, et al. Poly(L-lysine)-graftchitosan copolymers: synthesis, characterization, and gene transfection effect. Biomacromolecules. (2007) 8:1425-35. doi: 10.1021/bm060910u

28. Akaneya Y, Jiang B, Tsumoto T. RNAi-induced gene silencing by local electroporation in targeting brain region. J Neurophysiol. (2005) 93:594-602. doi: 10.1152/jn.00161.2004

29. Krutzfeldt J, Rajewsky N, Braich R, Rajeev KG, Tuschl T, Manoharan M, et al. Silencing of microRNAs in vivo with 'antagomirs'. Nature. (2005) 438:685-9. doi: 10.1038 /nature04303

30. McAllister DV, Allen MG, Prausnitz MR. Microfabricated microneedles for gene and drug delivery. Annu Rev Biomed Eng. (2000) 2:289-313. doi: 10.1146/annurev.bioeng.2.1.289

31. Davis HL, Whalen RG, Demeneix BA. Direct gene transfer into skeletal muscle in vivo: factors affecting efficiency of transfer and stability of expression. Hum Gene Ther. (1993) 4:151-9. doi: 10.1089/hum.1993.4.2-151

32. Corsi K, Chellat F, Yahia L, Fernandes JC. Mesenchymal stem cells, MG63 and HEK293 transfection using chitosan-DNA nanoparticles. Biomaterials. (2003) 24:1255-64. doi: 10.1016/S0142-9612(02)00507-0

33. Anderson DG, Akinc A, Hossain N, Langer R. Structure/property studies of polymeric gene delivery using a library of poly( $\beta$-amino esters). Mol Ther. (2005) 11:426-34. doi: 10.1016/j.ymthe.2004.11.015

34. Boussif O, Lezoualc'h F, Zanta MA, Mergny MD, Scherman D, Demeneix B, et al. A versatile vector for gene and oligonucleotide transfer into cells in culture and in vivo: polyethylenimine. Proc Natl Acad Sci USA. (1995) 92:7297-301. doi: 10.1073/pnas.92.16.7297

35. Mintzer MA, Simanek EE. Nonviral vectors for gene delivery. Chem Rev. (2009) 109:259-302. doi: 10.1021/cr800409e

36. Regnstrom K, Ragnarsson EG, Fryknas M, Koping-Hoggard M, Artursson P. Gene expression profiles in mouse lung tissue after administration of two cationic polymers used for nonviral gene delivery. Pharm Res. (2006) 23:475-82. doi: 10.1007/s11095-006-9563-7

37. Liu H, Slamovich EB, Webster TJ. Less harmful acidic degradation of poly(lacticco-glycolic acid) bone tissue engineering scaffolds through titania nanoparticle addition. Int J Nanomed. (2006) 1:541-5. doi: 10.2147/nano.2006.1.4.541

38. Pack DW, Hoffman AS, Pun S, Stayton PS. Design and development of polymers for gene delivery. Nat Rev Drug Discov. (2005) 4:581-93. doi: $10.1038 / \mathrm{nrd} 1775$

39. Cheung RC, Ng TB, Wong JH, Chan WY. Chitosan: an update on potential biomedical and pharmaceutical applications. Mar Drugs. (2015) 13:5156-86. doi: $10.3390 / \mathrm{md} 13085156$

40. Muxika A, Etxabide A, Uranga J, Guerrero P, de la Caba K. Chitosan as a bioactive polymer: processing, properties and applications. Int J Biol Macromol. (2017) 105(Pt. 2):1358-68. doi: 10.1016/j.ijbiomac.2017.07.087

41. Millner RW, Lockhart AS, Bird H, Alexiou C. A new hemostatic agent: initial life-saving experience with Celox (chitosan) in cardiothoracic surgery. Ann Thorac Surg. (2009) 87:e13-4. doi: 10.1016/j.athoracsur.2008.09.046

42. Paul P, Kolesinska B, Sujka W. Chitosan and Its Derivatives - Biomaterials with Diverse Biological Activity for Manifold Applications. Mini Rev Med Chem. (2019) 19:737-50. doi: 10.2174/1389557519666190112142735

43. Tang H, Zhang P, Kieft TL, Ryan SJ, Baker SM, Wiesmann WP, et al. Antibacterial action of a novel functionalized chitosan-arginine against Gram-negative bacteria. Acta Biomater. (2010) 6:2562-71. doi: $10.1016 /$ j.actbio.2010.01.002

44. Dai T, Tanaka M, Huang YY, Hamblin MR. Chitosan preparations for wounds and burns: antimicrobial and wound-healing effects. Expert Rev Anti Infect Ther. (2011) 9:857-79. doi: 10.1586/eri.11.59 
45. Ahmed TA, Aljaeid BM. Preparation, characterization, and potential application of chitosan, chitosan derivatives, and chitosan metal nanoparticles in pharmaceutical drug delivery. Drug Des Devel Ther. (2016) 10:483-507. doi: 10.2147/DDDT.S99651

46. Agnihotri SA, Mallikarjuna NN, Aminabhavi TM. Recent advances on chitosan-based micro- and nanoparticles in drug delivery. J Control Release. (2004) 100:5-28. doi: 10.1016/j.jconrel.2004.08.010

47. Lee WH, Loo CY, Young P, Traini D, Rohanizadeh R. The development and achievement of polymeric nanoparticles for cancer drug treatment. In: Sougata J, Subrata J, editors. Particulate Technology for Delivery of Therapeutics. India: Springer (2017). p. 25-82.

48. Zorzi GK, Carvalhob ELS, von Poser GL, Teixeira HF. On the use of nanotechnology-based strategies for association of complex matrices from plant extracts. Rev Brasilena Farmacogn. (2015) 25:426-36. doi: 10.1016/j.bjp.2015.07.015

49. Bayon-Cordero L, Alkorta I, Arana L. Application of solid lipid nanoparticles to improve the efficiency of anticancer drugs. Nanomaterials. (2019) 9:474. doi: 10.3390/nano9030474

50. Torchilin VP. Recent advances with liposomes as pharmaceutical carriers. Nat Rev Drug Discov. (2005) 4:145-60. doi: 10.1038/nrd1632

51. Li B, Lane LA. Probing the biological obstacles of nanomedicine with gold nanoparticles. Wiley Interdiscip Rev Nanomed Nanobiotechnol. (2019) 11:e1542. doi: 10.1002/wnan.1542

52. Che Marzuki NH, Wahab RA, Abdul Hamid M. An overview of nanoemulsion: concepts of development and cosmeceutical applications. Biotechnol Biotechnol Equip. (2019) 33:779-97. doi: 10.1080/13102818.2019.1620124

53. Yue J, Liu S, Wang R, Hu X, Xie Z, Huang Y, et al. Transferrin-conjugated micelles: enhanced accumulation and antitumor effect for transferrinreceptor-overexpressing cancer models. Mol Pharmaceut. (2012) 9:1919-31. doi: $10.1021 / \mathrm{mp} 300213 \mathrm{~g}$

54. Bu G, Maksymovitch EA, Geuze H, Schwartz AL. Subcellular localization and endocytic function of low density lipoprotein receptor-related protein in human glioblastoma cells. J Biol Chem. (1994) 269:29874-82.

55. Tan J-KY, Sellers DL, Pham B, Pun SH, Horner PJ. Non-viral nucleic acid delivery strategies to the central nervous system. Front Mol Neurosci. (2016) 9:108. doi: 10.3389/fnmol.2016.00108

56. Rosenblum D, Joshi N, Tao W, Karp JM, Peer D. Progress and challenges towards targeted delivery of cancer therapeutics. Nat Commun. (2018) 9:1410. doi: 10.1038/s41467-018-03705-y

57. An Z, Aksoy O, Zheng T, Fan QW, Weiss WA. Epidermal growth factor receptor and EGFRvIII in glioblastoma: signaling pathways and targeted therapies. Oncogene. (2018) 37:1561-75. doi: 10.1038/s41388-017-0045-7

58. Westphal M, Maire CL, Lamszus K. EGFR as a target for glioblastoma treatment: an unfulfilled promise. CNS Drugs. (2017) 31:723-35. doi: 10.1007/s40263-017-0456-6

59. Hegi ME, Diserens AC, Bady P, Kamoshima Y, Kouwenhoven MC, Delorenzi $\mathrm{M}$, et al. Pathway analysis of glioblastoma tissue after preoperative treatment with the EGFR tyrosine kinase inhibitor gefitinib-a phase II trial. Mol Cancer Ther. (2011) 10:1102-12. doi: 10.1158/1535-7163.MCT-11-0048

60. Danhier F, Messaoudi K, Lemaire L, Benoit J-P, Lagarce F. Combined anti-Galectin-1 and anti-EGFR siRNA-loaded chitosan-lipid nanocapsules decrease temozolomide resistance in glioblastoma: in vivo evaluation. Int J Pharmaceut. (2015) 481:154-61. doi: 10.1016/j.ijpharm.2015. 01.051

61. Van Woensel M, Mathivet T, Wauthoz N, Rosiere R, Garg AD, Agostinis $\mathrm{P}$, et al. Sensitization of glioblastoma tumor micro-environment to chemoand immunotherapy by Galectin-1 intranasal knock-down strategy. Sci Rep. (2017) 7:1217. doi: 10.1038/s41598-017-01279-1

62. Islam SU, Shehzad A, Ahmed MB, Lee YS. Intranasal delivery of nanoformulations: a potential way of treatment for neurological disorders. Molecules. (2020) 25:1929. doi: 10.3390/molecules25081929

63. Posadas I, Monteagudo S, Ceña V. Nanoparticles for brain-specific drug and genetic material delivery, imaging and diagnosis. Nanomedicine. (2016) 11:833-49. doi: 10.2217/nnm.16.15

64. Babu A, Amreddy N, Muralidharan R, Pathuri G, Gali H, Chen A, et al. Chemodrug delivery using integrin-targeted PLGA-Chitosan nanoparticle for lung cancer therapy. Sci Rep. (2017) 7:14674. doi: 10.1038/s41598-017-15012-5
65. Lakkadwala S, Singh J. Co-delivery of doxorubicin and erlotinib through liposomal nanoparticles for glioblastoma tumor regression using an in vitro brain tumor model. Colloids Surf B Biointerfaces. (2019) 173:27-35. doi: 10.1016/j.colsurfb.2018.09.047

66. Sahin A, Yoyen-Ermis D, Caban-Toktas S, Horzum U, Aktas Y, Couvreur $\mathrm{P}$, et al. Evaluation of brain-targeted chitosan nanoparticles through bloodbrain barrier cerebral microvessel endothelial cells. J Microencapsul. (2017) 34:659-66. doi: 10.1080/02652048.2017.1375039

67. Sharma AK, Gupta L, Sahu H, Qayum A, Singh SK, Nakhate KT, et al. Chitosan engineered PAMAM dendrimers as nanoconstructs for the enhanced anti-cancer potential and improved in vivo brain pharmacokinetics of temozolomide. Pharm Res. (2018) 35:9. doi: 10.1007/s11095-017-2324-y

68. Raja MA, Katas H, Jing Wen T. Stability, intracellular delivery, and release of sirna from chitosan nanoparticles using different cross-linkers. PLoS ONE. (2015) 10:e0128963. doi: 10.1371/journal.pone.0128963

69. Gwak SJ, Jung JK, An SS, Kim HJ, Oh JS, Pennant WA, et al. Chitosan/TPP-hyaluronic acid nanoparticles: a new vehicle for gene delivery to the spinal cord. J Biomater Sci Polym Ed. (2012) 23:1437-50. doi: 10.1163/092050611X584090

70. Ying M, Wang S, Sang Y, Sun P, Lal B, Goodwin CR, et al. Regulation of glioblastoma stem cells by retinoic acid: role for Notch pathway inhibition. Oncogene. (2011) 30:3454-67. doi: 10.1038/onc.2011.58

71. Mourya VK, Inamdar NN. Trimethyl chitosan and its applications in drug delivery. J Mater Sci Mater Med. (2009) 20:1057-79. doi: $10.1007 / \mathrm{s} 10856-008-3659-\mathrm{z}$

72. Jaiswal M, Dudhe R, Sharma PK. Nanoemulsion: an advanced mode of drug delivery system. 3 Biotech. (2015) 5:123-7. doi: 10.1007/s13205-014-0214-0

73. Chandy T, Das GS, Rao GH. 5-Fluorouracil-loaded chitosan coated polylactic acid microspheres as biodegradable drug carriers for cerebral tumours. $J$ Microencapsul. (2000) 17:625-38. doi: 10.1080/026520400417676

74. Colombo M, Figueiro F, de Fraga Dias A, Teixeira HF, Battastini AMO, Koester LS. Kaempferol-loaded mucoadhesive nanoemulsion for intranasal administration reduces glioma growth in vitro. Int J Pharm. (2018) 543:21423. doi: 10.1016/j.ijpharm.2018.03.055

75. Letchford K, Burt $\mathrm{H}$. A review of the formation and classification of amphiphilic block copolymer nanoparticulate structures: micelles, nanospheres, nanocapsules and polymersomes. Eur $J$ Pharmaceut Biopharmaceut. (2007) 65:259-69. doi: 10.1016/j.ejpb.2006.11.009

76. Jeong YI, Kim S-H, Jung T-Y, Kim I-Y, Kang S-S, Jin YH, et al. Polyion complex micelles composed of all-trans retinoic acid and poly (ethylene glycol)-grafted-chitosan. J Pharmaceut Sci. (2006) 95:2348-60. doi: 10.1002/jps.20586

77. Wang G, Wang JJ, Tang $\mathrm{XJ}$, Du L, Li F. In vitro and in vivo evaluation of functionalized chitosan-Pluronic micelles loaded with myricetin on glioblastoma cancer. Nanomedicine. (2016) 12:1263-78. doi: 10.1016/j.nano.2016.02.004

78. Agrawal P, Sonali, Singh RP, Sharma G, Mehata AK, Singh S, et al. Bioadhesive micelles of d-alpha-tocopherol polyethylene glycol succinate 1000: Synergism of chitosan and transferrin in targeted drug delivery. Colloids Surf B Biointerfaces. (2017) 152:277-88. doi: 10.1016/j.colsurfb.2017.01.021

79. Agrawal P, Singh RP, Sonali, Kumari L, Sharma G, Koch B, et al. TPGS-chitosan cross-linked targeted nanoparticles for effective brain cancer therapy. Mater Sci Eng C Mater Biol Appl. (2017) 74:167-76. doi: 10.1016/j.msec.2017.02.008

80. Bruinsmann FA, Pigana S, Aguirre T, Dadalt Souto G, Garrastazu Pereira G, Bianchera A, et al. Chitosan-coated nanoparticles: effect of chitosan molecular weight on nasal transmucosal delivery. Pharmaceutics. (2019) 11:86. doi: 10.3390/pharmaceutics11020086

81. Erickson AE, Lan Levengood SK, Sun J, Chang FC, Zhang M. Fabrication and characterization of chitosan-hyaluronic acid scaffolds with varying stiffness for glioblastoma cell culture. Adv Healthc Mater. (2018) 7:e1800295. doi: 10.1002/adhm.201800295

82. Kuo YC, Wang LJ, Rajesh R. Targeting human brain cancer stem cells by curcumin-loaded nanoparticles grafted with anti-aldehyde dehydrogenase and sialic acid: Colocalization of ALDH and CD44. Mater Sci Eng C Mater Biol Appl. (2019) 102:362-72. doi: 10.1016/j.msec.2019.04.065

83. Kutlu C, Cakmak AS, Gumusderelioglu M. Double-effective chitosan scaffoldPLGA nanoparticle system for brain tumour therapy: in vitro study. J Microencapsul. (2014) 31:700-7. doi: 10.3109/02652048.2014.913727 
84. Saboktakin MR, Tabatabaie RM, Maharramov A, Ramazanov MA. Synthesis and characterization of $\mathrm{pH}$-dependent glycol chitosan and dextran sulfate nanoparticles for effective brain cancer treatment. Int J Biol Macromol. (2011) 49:747-51. doi: 10.1016/j.ijbiomac.2011.07.006

85. Sedeky AS, Khalil IA, Hefnawy A, El-Sherbiny IM. Development of core-shell nanocarrier system for augmenting piperine cytotoxic activity against human brain cancer cell line. Eur J Pharm Sci. (2018) 118:103-12. doi: 10.1016/j.ejps.2018.03.030

86. Trapani A, Denora N, Iacobellis G, Sitterberg J, Bakowsky U, Kissel T. Methotrexate-loaded chitosan- and glycol chitosan-based nanoparticles: a promising strategy for the administration of the anticancer drug to brain tumors. AAPS Pharm Sci Tech. (2011) 12:1302-11. doi: 10.1208/s12249-011-9695-x

87. Varan C, Bilensoy E. Cationic PEGylated polycaprolactone nanoparticles carrying post-operation docetaxel for glioma treatment. Beilstein $J$ Nanotechnol. (2017) 8:1446-56. doi: 10.3762/bjnano.8.144
88. Fu S, Xia J, Wu J. Functional chitosan nanoparticles in cancer treatment. J Biomed Nanotechnol. (2016) 12:1585-603. doi: 10.1166/jbn. 2016.2228

Conflict of Interest: The authors declare that the research was conducted in the absence of any commercial or financial relationships that could be construed as a potential conflict of interest.

Copyright (C) 2020 Lara-Velazquez, Alkharboosh, Norton, Ramirez-Loera, Freeman, Guerrero-Cazares, Forte, Quiñones-Hinojosa and Sarabia-Estrada. This is an openaccess article distributed under the terms of the Creative Commons Attribution License (CC BY). The use, distribution or reproduction in other forums is permitted, provided the original author(s) and the copyright owner(s) are credited and that the original publication in this journal is cited, in accordance with accepted academic practice. No use, distribution or reproduction is permitted which does not comply with these terms. 